\title{
Síndrome X frágil: Desarrollo e intervención del lenguaje escrito
}

\section{Fragile X syndrome: Development and intervention of written language}

\author{
Ma Paz Fernández L. ${ }^{1}$, Aníbal Puente F. y Ma Teresa Ferrando L. ${ }^{3}$
}

\begin{abstract}
Introduction: The article aims to provide an up-to-date review of the difficulties children and adolescents with fragile X disorders have in school. Fragile X syndrome (FXS) is the most frequent cause of hereditary intellectual disability, as well as being a common cause of learning disorders and behavioural problems. It is characterised by very specific physical and behavioural phenotypes. FXS is caused by a mutation in the FMR1 gene (Fragile X Mental Retardation) located at the bottom end of the X chromosome at Xq27.3. This gene mutation produces an expansion in the number of CGG (Cytosine, Guanine, Guanine) triplet repetitions. The full mutation causes a state of hypermethylation that inhibits gene expression. Analysis of fragile X syndrome represents a good model for determining the relationship between genes and behaviour. Development: There are many grey areas in the area of reading and writing in the fragile $X$ population. Very few basic and applied research studies are available and they mostly assume behavioural patterns derived from comparable populations such as Down syndrome, Autism, and Williams syndrome. Conclusion: The main problems FXS children have with reading and writing stem from language and motor disorders and difficulties with sensorial integration. Our work aims to broaden and to systemize some of the aspects which we consider key to improving educational practice and to overcome reading deficiencies. In this direction, we highlight some programmatic proposals and methodological strategies which should be explored for their educational value and practical effectiveness.
\end{abstract}

Key words: Fragile $X$ syndrome, reading, writing, strategies, learning, teaching, intellectual disability.

Rev Chil Neuro-Psiquiat 2010; 48 (3): 219-231

Recibido: 08/01/2010

Aceptado: 07/09/2010

1 Profesora Asociada, Facultad de Psicología, Departamento de Psicología Básica II (Procesos Cognitivos), Universidad Complutense de Madrid.

2 Catedrático de Psicología, Facultad de Psicología, Departamento de Psicología Básica II (Procesos Cognitivos), Universidad Complutense de Madrid.

3 Médico del Servicio de Neuropediatría, Hospital Quirón de Madrid, Profesora Asociada del Colegio Universitario La Salle. 


\section{Introducción}

$\mathrm{C}^{\mathrm{l}}$ síndrome $\mathrm{X}$ frágil (SXF) fue descrito por Eprimera vez por Martín y Bell ${ }^{1}$, pero los verdaderos descubrimientos sucedieron después, cuando Lubs $^{2}$ encontró una alteración citogenética ("cromosoma marcador") en niños con discapacidad intelectual, y diez años más tarde, cuando Sutherland ${ }^{3}$ localizó los sitios frágiles del cromosoma X. Otro hito insoslayable es el descubrimiento del gen FMR1, secuenciado en 1991 por un consorcio internacional dirigido por el Dr. Verkerk. De modo significativo hay que señalar que los sitios frágiles mencionados no son detectables a través del cariotipo convencional.

El X frágil es la segunda causa genética de discapacidad intelectual, siendo la primera de discapacidad intelectual hereditario; la cifra estimada de portadores es de 1:4.000 varones y 1:6.000 mujeres ${ }^{4}$. La anomalía es debida a una mutación genética del ADN que afecta tanto a las células sexuales (óvulos y espermatozoides) como a otros tipos de células de nuestro organismo. El SXF obedece a un defecto molecular consistente en una cantidad aumentada de repeticiones de la tripleta CGG (Citosina, Guanina, Guanina) en una región del gen FMR1 (Fragile X Mental Redartation 1) situada en la porción terminal del brazo largo del cromosoma $\mathrm{X}^{5}$. El gen FMR1 codifica una proteína llamada FMRP, que está ausente o muy disminuida, en las personas afectadas. Esta proteína es necesaria para la poda de excesos de botones sinápticos, durante la hodogénesis, y para el desarrollo del tejido conjuntivo. El déficit o ausencia de proteína tiene, por tanto como consecuencia, un exceso de sustancia blanca, base del fenotipo cognitivo-conductual, y una disfunción de todos los sistemas derivados del tejido conjuntivo, de donde se deriva la rica semiología general que pueden presentar los afectados ${ }^{6,7}$.

En la población general el número de repeticiones CGG, oscila entre 5 y 50; el aumento del número de repeticiones entre 50 y 200 corresponde al estado de portador; en esta situación la tripleta se vuelve inestable y tiende a expandirse, con lo que en un siguiente paso generacional, si aumenta por encima de 200 repeticiones, la situación es de mu- tación completa; este genotipo se corresponde con un fenotipo cognitivo-conductual y en ocasiones físico que define la clínica del síndrome $\mathrm{X}$ frágil ${ }^{8,9}$. El hecho de que el SXF sea hereditario implica un riesgo para los demás miembros de la familia del individuo afectado. El predominio de varones afectados con el síndrome $\mathrm{X}$ frágil y la existencia de algunas mujeres afectadas hizo pensar inicialmente que el SXF seguía una herencia dominante ligada al X. Sin embargo, pronto se comprobó que muchas familias no encajaban en este tipo de herencia mendeliana convencional ya que dependía del tipo de mutación genética que presenta cada paciente. Los varones afectados tienen la llamada "mutación completa", mientras que las madres portadoras tienen una "premutación", que al pasar a su hijo puede "expandirse a mutación completa" ${ }^{10}$. La mutación completa se encuentra en todos los varones SXF, mientras que sólo alrededor de un $65 \%$ de mujeres con mutación completa presenta déficit intelectual. Otro dato interesante es que aproximadamente un $40 \%$ de individuos con mutación completa son también portadores de células con premutación ${ }^{11}$. La premutación se produce en el $80 \%$ de las mujeres portadoras asintomáticas y en el $100 \%$ de los hombres portadores.

El X frágil casi siempre se manifiesta en un discapacidad intelectual, de leve a moderado (muy raras veces severo). Los síntomas más precoces son el retraso en adquisición de hitos madurativos neurológicos, tanto motores como del lenguaje, constituyendo el retraso en la maduración del lenguaje el síntoma más frecuente de primera consulta. Un rasgo constante en los $\mathrm{X}$ frágil es la hiperactividad ${ }^{12}$ que a su vez suele ir acompañado de lapsos cortos de atención y conducta táctil defensiva. Por último, no hay que olvidar además, que el SXF puede manifestarse también en las niñas y mujeres, pero con una gran variabilidad. Son raros pero existen, los casos con fenotipo semejantes a los niños ${ }^{2}$, pero lo más frecuente es que no haya ningún rasgo físico característico, manifestando sólo un RM ligero, con trastornos de conducta (aislamiento, angustia social, timidez) y problemas sobre todo en el lenguaje y en el área de las matemáticas. El fenotipo físico (macrocefalia, orejas grandes y despegadas, facies 
alargada, prognatismo y macroorquia) se presenta en varones con mutación completa, pero hay que tener en cuenta que hasta la pubertad puede pasar desapercibido e incluso no manifestar el fenotipo facial a pesar de padecer la mutación completa (10$20 \%$ de los casos).

Como se ha señalado, la frecuencia estimada, en todo el mundo, supone que 1:4.000 varones y 1:6.000 mujeres están afectadas por la enfermedad ${ }^{4}$. En el caso de portadores se estima 1:260 mujeres y 1:800 hombres ${ }^{13,14}$. Estas cifras extrapoladas a nuestro país pueden hacer concluir que existirían alrededor de 10.000 afectados y 100.000 portadores aunque en España no existe un censo que contemple la totalidad de los $\operatorname{casos}^{15}$. En España, el trabajo más extenso con una población clínica diagnosticada, ha sido efectuado por el grupo del Dr. Artigas-Pallarés a partir de datos obtenidos de 106 niños afectos del síndrome, en colaboración con diferentes Asociaciones del Síndrome ${ }^{16}$.

Uno de los aspectos menos conocido del X frágil es el relacionado con la lectura y la escritura. Existe poca investigación conocida a nivel internacional ${ }^{17} \mathrm{y}$ en el ámbito nacional es muy limitada. La mayoría de la información disponible procede de la literatura de niños y adultos con síndrome Down $^{18}$. La escasa información relacionada con la lectura de los X frágil plantea la existencia de algunos patrones similares en el desarrollo y progreso del comportamiento lector de los $\mathrm{X}$ frágil y los SD. El conocimiento, por ende, que poseemos de la lectura de los SD se puede aplicar en líneas generales a los $\mathrm{X}$ frágil y las recomendaciones para intervenir los procesos de alfabetización parecen también pertinentes en este momento ${ }^{19}$.

\section{Objetivos}

La propuesta que desarrollamos pretende: a) Ordenar la escasa y dispersa literatura existente y analizar los factores que explican las disfunciones lectoras de los X frágil tomando en consideración la información derivada de las investigaciones ejecutadas en los países anglosajones, principalmente; b) Ofrecer información empírica a la comunidad científica hispanohablante y a los grupos de profe- sionales dedicados a la educación, rehabilitación y tratamiento de los X frágil; c) Promover entre los especialistas investigaciones básicas y aplicadas en relación con el comportamiento lector y escritor; d) Aliviar la preocupación y ansiedad de las familias y asociaciones ante el bajo rendimiento en lectura/escritura de estos niños, sabiendo que existen medios tecnológicos y metodologías que ofrecen posibilidades para modificar las perspectivas de futuro y de mejorar la independencia y autonomía que la lectura representa para el colectivo con $\mathrm{X}$ frágil.

\section{Metodología}

Una de las características de la literatura relacionada con el lenguaje de los X frágil es la dispersión. Hasta fechas recientes apenas se conocían datos consistentes que nos permitieran extraer conclusiones firmemente asentadas. Los datos conocidos procedían de observaciones clínicas con escasa representación de la población. Sin embargo, en la década de los noventa hemos acumulado datos suficientes para elaborar una síntesis integrada y una revisión actualizada. Uno aspecto crucial metodológico a considerar fue la definición de el/los grupo/s de comparación del perfil lingüístico de los $\mathrm{X}$ frágil. Estos grupos de referencia fueron niños y adolescentes con síndrome Down y niños normales de similares características en edad, sexo y nivel intelectual. El análisis y revisión de las investigaciones se ha concentrado en aspectos relacionados con el lenguaje escrito (lectura y escritura), destacando su adquisición, evolución e intervención. Las áreas del lenguaje examinadas fueron: habilidades receptivas y expresivas, habilidades pragmáticas, componentes contextuales, procesos de comunicación y sobre todo las habilidades necesarias para el rendimiento académico en tareas escolares. La revisión de la literatura abarcó un amplio aspecto de fuentes de información, todas ellas relevantes con el tema que nos ocupa. En ella se ha incluido artículos científicos de la base datos PUBMED con entradas: speech \& language development \& intervention in Fragile $\mathrm{X}$ syndrome of children and adolescents de los últimos 10 años. Después se buscaron los artículos 
relevantes de fechas anteriores que aparecen en las referencias de los artículos más recientes. En las revistas y libros fuente más importantes encontramos las siguientes: Journal of Neurology, Neurosurgery and Psychiatry, Journal of Mental Deficiency Research, American Journal of Medical Genetics, Human Genetics, New England Journal of Medicine, Revista de Neurología, Cell, Mental Retardation and Developmental Disabilities Research Reviews, American Journal on Mental Retardation, Journal of Speech, Language and Hearing Research, etc. Se resumen y sistematizan los hallazgos más importantes.

\section{El lenguaje y los problemas de lectura}

Una explicación recurrente de las dificultades lectoras de los $\mathrm{X}$ frágil está relacionada con los problemas de lenguaje de estas personas. Las dificultades de lenguaje de los niños con $\mathrm{X}$ frágil fluctúan entre un habla entrecortada y problemas graves relacionados con las habilidades lingüísticas básicas. Entre los problemas más severos podemos destacar: la incapacidad de pronunciar palabras de forma clara, la dificultad para escribir y usar la gramática de manera correcta y la torpeza para comunicarse de manera significativa ${ }^{20,21}$.

En algunos X frágil, los problemas son más severos. Muchos comienzan a hablar más tarde de lo esperado: producen las primeras palabras a la edad de cuatro años y no hablan hasta los seis u ocho años $^{22,23}$. Otros, de forma eventual, mantienen un lenguaje no-verbal durante toda la vida. Para estos niños no-verbales existen una variedad de dispositivos que se puede usar (fotos, esquemas, ordenador, etc.) que pueden ayudarles a comunicarse. El uso de fotos, ordenador, lengua de señas y gestos también se pueden utilizar para todos los niños $\mathrm{X}$ frágil antes de comenzar a hablar ${ }^{24}$.

Las niñas con X frágil muy raramente presentan problemas con el lenguaje hablado. En efecto, muchas de ellas manejan un vocabulario adecuado y usan también la gramática de acuerdo a su edad lo que les permite aprender a leer y escribir. Sin embargo, la ansiedad social y la timidez les impiden comunicarse de forma apropiada ${ }^{25}$. Algunas niñas usan muchos circunloquios y formas de habla desorganizada o se van fuera del tema de la conversación.

\section{Eficiacia del entorno escolar}

De forma reciente, se viene manifestando una corriente que considera el entorno escolar como una de las variables que puede influir en el rendimiento académico de los $\mathrm{X}$ frágil. Un interesante estudio desarrollado por Symons, Clarke \& Robersts $^{26}$ concluye que el compromiso de los X frágil con las tareas escolares está muy relacionado con la calidad ambiental, la instrucción de los profesores, las aulas y la motivación. Los autores observaron que la manera cómo los profesores estructuran y organizan el entorno es más importante que los aspectos específicos de los chicos con $\mathrm{X}$ frágil; tales como la severidad de la enfermedad, la medicación usada o el diagnóstico. Esta misma conclusión ha sido validada por Braden ${ }^{24,27}$ que sugiere que la mejor práctica para ser usada en la escuela con los niños afectados de $\mathrm{X}$ frágil es proporcionar un entorno de aprendizaje que permita desarrollar el plan de estudios con el menor número de interrupciones posibles y con profesores menos preocupados por los niveles funcionales de los niños y los diagnósticos complejos.

Los maestros de aula y especialistas proponen que una educación "apropiada" requiere acomodación y adaptación específicas acorde con la condición de los estudiantes. Por ejemplo, los $\mathrm{X}$ frágil tienen un agudo sentido de pertenencia, especialmente cuando estos niños perciben que su rendimiento no va al mismo ritmo que el de sus compañeros de clase. Este reconocimiento de auto-exclusión del grupo puede tener un impacto importante en su compromiso y comportamiento académico.

\section{Lectura de las niñas con $\mathrm{X}$ frágil}

Muchas niñas con SXF decodifican muy bien y poseen buenas habilidades de comprensión de la lectura ${ }^{19,28}$. Algunas de ellas rinden mejor cuando hacen interpretaciones literales durante la lectura 
que cuando tienen que hacer inferencias. En general, se puede afirmar que la lectura de las chicas constituye una de las habilidades cuyo perfil es el esperado para su edad y desarrollo intelectivo. Algunos especialistas e investigadores ${ }^{29,39}$ señalan que éste es uno de los dominios relativamente fuertes de las niñas; algo que no ocurre en los niños SXF. Los chicos muestran un rango amplio de habilidades lectoras: existe un grupo pequeño que son clasificados como no lectores; otro grupo, relativamente amplio, que son capaces de leer palabras aisladas, frases y textos cortos; y un tercer grupo, pequeño en número, que alcanza un nivel suficiente para leer capítulos cortos de un libro, revistas y periódicos ${ }^{31}$.

\section{Método global de enseñanza}

A los maestros que enseñan a los niños con SXF, se les recomienda utilizar un método de lectura visual, apoyándose en el uso de la palabra "como un todo" (método global). A partir de las observaciones de los clínicos y maestros, hoy sabemos que este método resulta más eficaz que otros, como el silábico o el fonológico. Esta impresión clínica no siempre ha sido confirmada con los datos de la investigación científica. Recientemente, JohnsonGlenberg ${ }^{32}$ comparó un grupo de niños SXF con otro grupo de niños normales, ambos grupos eran homogéneos en las habilidades decodificadoras. La investigadora encontró que los chicos SXF mostraban mayores dificultades que los otros niños a la hora de leer no-palabras. La razón que explica el bajo rendimiento se debe a que los SXF carecen de un adecuado desarrollo del componente fonológico, particularmente la conciencia fonológica. Esta deficiencia es uno de los elementos que dificulta que los SXF no recuerden las secuencias verbales mientras leen y no comprendan frases y textos $\operatorname{cortos}^{33}$.

Los SXF prelectores y lectores tienen serios problemas con muchas áreas auditivas. Aspectos auditivos tales como discriminar sonidos, reconocer sonidos fuera de las palabras, mezclar sonidos para construir palabras, silabear y reconocer si dos palabras riman. Todas estas tareas resultan muy difíciles para los niños y adolescentes con $\mathrm{SXF}^{29}$. Si estos chicos no son capaces de separar los sonidos constituyentes de una palabra o mezclar sonidos para formar palabras completas, significaría que para ellos es muy difícil adquirir la lectura a través del método fonético. Los niños podrían reconocer la palabra /Sol/ y relacionarla con el significado, pero no podrían relacionar las letras en la palabra con los correspondientes sonidos. La alternativa que se plantea al método fonético y silábico es el método de la palabra global. Una observación de interés pedagógico expresada por maestros de lenguaje y logopedas es que el método global es más eficaz que el método fonético para enseñar lectura a los $\mathrm{SXF}^{24,27,34}$. Inicialmente conviene enseñar palabras que el niño tiene en su vocabulario receptivo y después seleccionar palabras que sean visual y fonéticamente diferentes.

Algunas orientaciones prácticas con los SXF en relación con la lectura son las siguientes: imprimir las palabras de modo claro, con letras bien formadas, de modo que los niños las puedan ver, reconocer su forma, asociar la forma con los sonidos y pronunciarlas si fuera posible. El acompañamiento con refuerzo sensorial es algo que muchos especialistas recomienda en el entrenamiento lector en la población general y de modo especial con los SXF $^{35,36}$. La técnica Palabra-Objeto (por ejemplo: silla, ventana, libro y otros objetos del aula) con sus correspondientes asociaciones ha resultado una técnica altamente eficaz ${ }^{31}$. Esta técnica de asociación de dibujos a nombres, leer nombres de las señales del metro o la ciudad, leer nombres en cuadros o viñeta, etc se denomina Logo Reading System, que padres y maestros consideran un excelente programa para ayudar a desarrollar las habilidades de lectura en niños con $\mathrm{X}$ frágil, autismo y otras alteraciones del desarrollo. Johnson y Myklebust ${ }^{37}$ también insisten en usar el método visual para leer y escribir frases cortas como /pelota grande/, /pelota pequeña/, /pelota azul/. Posteriormente, la lectura se puede expandir a la lectura de órdenes como /pon el libro en la mesa/. Finalmente, el progreso debe incluir la lectura de pequeñas historias; por ejemplo, las experiencias que ocurren dentro del 
aula de clase, acompañadas de dibujos y estímulos que faciliten la comprensión. Estas actividades promueven la comprensión de las tareas a medida que los niños hablan de sus actividades y planes. No se puede olvidar que a los SXF se les facilita mucho la tarea cuando los materiales de lectura son claros, bien espaciados y no muy densos.

\section{Lectura de no-palabras}

La lectura de no-palabras (/mosa/) es una tarea que resulta más difícil que la lectura de palabras, sobre todo, si estas últimas son familiares y concretas; pero en el caso de los SXF, la diferencia es mucho mayor. Esta diferencia se ha podido comprobar al aplicar varias pruebas ${ }^{38,39}$. Uno de los factores que mejor explica la diferencia es el componente fonológico y su procesamiento ${ }^{40}$. Las pruebas a favor del peso del procesamiento fonológico en las tareas de lectura son extensas y confirmatorias en la literatura. La constatación de la importancia de este factor en niños con déficit intelectual también son abundantes ${ }^{41}$. Otros investigadores plantean que entre lectura y conciencia fonológica existe un efecto recíproco ${ }^{42}$. La adquisición de la lectura requiere el dominio de habilidades fonológicas básicas y a medida que la lectura se incrementa, las habilidades fonológicas se consolidan más y más. ¿Cuál de los elementos de la relación es más importante? Todavía no contamos con apoyo científico suficiente para saberlo con certeza. De lo que sí estamos seguros es que, si a los niños en el nivel preescolar se les entrena intensamente en conciencia fonológica, estos alumnos obtienen un mejor rendimiento en las medidas de lectura que aquéllos alumnos que no recibieron el entrenamiento ${ }^{43}$.

\section{Entrenamiento en conciencia fonológica}

Kumin $^{44}$, propone algunos ejemplos de tareas que sirven para el entrenamiento fonológico en niños con SXF y SD.

Estímulos fonéticos iniciales: Se proporciona el primer sonido de la palabra. Por ejemplo, si se quiere que el niño pronuncie la palabra "Colonial", diga " $\mathrm{K}, \mathrm{K}, \mathrm{K}$ ”, como estímulo fonético inicial.

Palabras-estímulo que riman: Se presenta a los lectores una palabra-estímulo que rima con otra, con el propósito de ayudar a que el niño recuerde la palabra. Por ejemplo, la palabra-estímulo es "Ana", y el niño tiene que decir "La rana es amiga de__ "y el espacio se debe rellenar con "Ana".

Palabras-estímulo asociadas: Se sugiere una palabra conectada de alguna manera al significado de otra palabra. Por ejemplo, se puede decir "Lápiz y ___ _ con la idea de ayudar al niño a asociar significados se le presenta la palabraestímulo "Papel" para que complete la frase. Las palabras opuestas como estímulos se usan con frecuencia; por ejemplo, "Arriba y___, "Frío y__ "

Palabras-estímulo en secuencia: Se ofrece una palabra-estímulo que podría aparecer en una secuencia. Esta actividad es parecida a la tarea de completar preguntas, por ejemplo se plantea la palabra "teléfono" y a continuación la secuencia para ser completada "Habla por

Estímulos visuales: Esta técnica puede adoptar diversos procedimientos.

- Estímulos escritos (usar la palabra, parte de la palabra, o el sonido inicial de una consonante para producir un estímulo impreso).

- Estímulos pictóricos (un dibujo o una fotografía se asocia con la palabra o la imagen del objeto).

- Iconos como estímulos (un símbolo que podría evocar una palabra o concepto, tal como un ojo para recordar a un niño que debe mirar al hablante).

- Estímulos en color para facilitar el aprendizaje (usar materiales con diferentes colores para señalar lo que hay que destacar en una tarea de estudio; usar marcadores fluorescentes, etc para asistir al niño en la tarea de aprendizaje).

Estímulos gestuales: Usar una señal manual o un apuntador como señal que puede evocar la palabra o el concepto; por ejemplo, levantar un dedo como señal de silencio o mantener levantadas las dos manos para solicitar ayuda. 


\section{Comprensión lectora}

Las metas de la lectura deberían estar determinadas por el nivel cognitivo de los SXF. En el caso de los niños que están comenzando la adquisición de la lectura, la meta podría girar en torno al entrenamiento de la lectura funcional ${ }^{18}$. Como sabemos, estos chicos eventualmente participan con sus padres en las compras y en las actividades de cocina. En estos casos, leer la lista del mercado, o leer el nombre de los productos, o repetir los pasos para la elaboración de un plato de comida son ejemplos de lectura funcional. La lectura funcional ofrece una extraordinaria oportunidad para que los niños mejoren su autonomía y su independencia. Las habilidades para leer e interpretar horarios e itinerarios pueden ayudarlos a usar el transporte público por sí mismo y conocer los programas que más les interesa de la TV. Igualmente ellos podría leer títulos de películas o señales que indican peligro en los edificios públicos. Todas estas tareas son ejemplos ilustrativos de lectura funcional, que tantos beneficios aportan al colectivo de SXF.

Para aquellos niños y adolescentes que aspiran a dominar la lectura con un nivel más alto y expandido es necesario entrenarlos en el uso de estrategias más exigentes para lograr la comprensión. Si los niños todavía mantienen dificultades para discriminar sonidos, la siguiente meta de lectura podría ser trabajar el significado de los morfemas. Un ejemplo de tareas siguiendo esta línea de actuación consistiría en separar la palabra en sílabas y reconocer el significado de los prefijos y/o sufijos como un eficaz método para expandir el vocabulario. Johnson y Mykleust ${ }^{37}$ plantean que no se usen líneas entre sílabas puesto que se puede distorsionar la imagen de la palabra y confundir. Una técnica más adecuada es utilizar espacios entre las partes de una palabra, de este modo, se ayuda a focalizar la visión de los niños y se facilita el escrutinio de las partes.

No es raro encontrar que algunos niños con SXF puedan ser hiperléxicos con mejor habilidad para decodificar palabras que para comprender$\operatorname{las}^{45}$. La comprensión literal puede ser otra meta, usando como base preguntas del tipo ¿Qu? acerca del texto (por ejemplo: ¿Qué hizo Juan? ¿Quién es su amigo? ¿Qué lleva en la mano?). También se puede entrenar la habilidad para realizar inferencias a partir del texto (por ejemplo: ¿Qué piensas que ocurrirá después? ¿Cómo se sentirá la madre?). Las actividades de comprensión se pueden alternan con otras que permitan al lector ir hacia atrás en el texto y aquéllas que requieren del lector memorizar y contestar preguntas. Los trabajos escritos, tales como contestar preguntas, deben ser muy simples, con la finalidad de asegurarse que las dificultades de escritura no interfieran con la habilidad para contestar preguntas que midan comprensión. Una forma de medir la comprensión es rellenar espacios en blanco, marcar con un círculo la respuesta correcta o comenzar la respuesta en voz alta. Las actividades de lectura y escritura deben examinarse con sumo cuidado para determinar si las dificultades derivan de una mala comprensión de lectura o son problemas de escritura.

\section{Lenguaje escrito}

El lenguaje escrito representa el nivel más alto del lenguaje $\mathrm{e}^{18}$. Las actividades de escritura exigen del niño un desarrollo cognitivo suficiente para comprender la tarea, mantener intacto el lenguaje receptivo, haber desarrollado las habilidades básicas de lectura, contar con habilidades motoras finas y memoria visual suficiente para deletrear, secuenciar letras, palabras e ideas. Las personas con SXF (particularmente niños) pueden sufrir déficit en alguna de estas habilidades o pueden encontrarse con problemas en todas ellas.

El lenguaje escrito requiere seguir instrucciones. Las destrezas para la escritura hay que enseñarlas, no se aprenden de manera espontánea como ocurre con otras, como es el caso del lenguaje. En los niños con SXF, el lenguaje receptivo y las habilidades de lectura deben entrenarse antes y durante las clases de escritura. El lenguaje escrito puede estar muy limitado para aquellos casos con déficit cognitivos moderados o severos ${ }^{46}$. Cuando esto sucede, puede ser más productivo reducir las actividades de escritura y dedicar más tiempo a reforzar otras áreas de aprendizaje. Como antes se ha indicado, la 
mejor forma de medir la comprensión escrita sería: marcar con un círculo la respuesta correcta, hacer un dibujo, contestar de forma verbal, o escribir con una palabra. Como actividades específicas de escritura podemos sugerir la escritura y firma del nombre o cualquier otra información importante para ellos (por ejemplo: dirección, nombre del colegio, nombre de los padres y hermanos).

\section{Alteraciones visomotoras de la escritura}

Algunos niños con SXF manifiestan problemas de carácter visual y motor cuando realizan tareas de escritura y dibujo ${ }^{19}$. Otros tienen dificultades para copiar, un problema que se conoce como disgrafía. De modo similar a la dispraxia para el habla, la disgrafía involucra dificultades en la planificación y ejecución motora.

Los terapeutas ocupacionales y lo maestros de educación especial necesitan observar y trabajar la postura, la forma de agarrar el lápiz, y la posición del papel. Aquellos chicos que tienen problemas motores es posible que necesiten estrategias kinestésicas, trazar las letras en arena de playa, y maestros que les enseñen técnicas de cómo agarrar el lápiz. Los niños prefieren trabajar mejor con materiales como plantillas, plastilinas, laberintos simples, actividades de trazado, juegos con puntos $y$ símbolos ${ }^{35}$. Una estrategia que se ha revelado muy eficaz es manejar el teclado del ordenador, esta estrategia ayuda mucho a los chicos a escribir con más claridad, a espaciar las palabras y a corregir los errores.

\section{Formulación de oraciones y sintaxis}

La sintaxis es un área muy difícil para los SXF y SD porque es abstracta y compleja ${ }^{47,48}$. Muchas niñas y algunos niños son capaces de escribir oraciones, párrafos y pasajes más extensos ${ }^{49}$. El objetivo se debe centrar en torno a la formulación de las oraciones, comenzando con oraciones sencillas, en las que los niños tienen que llenar un espacio en blan- co. De forma gradual se les puede dar el comienzo de una oración para que él la complete. La escritura de párrafos puede requerir mucho entrenamiento ${ }^{50}$. El comienzo sería una oración que incluya el tema y después continuar con tres oraciones que expliquen y apoyen la oración-tema. Los temas han de ser muy concretos y haberlos discutido con los niños y adolescentes antes de empezar a escribir. Hay que trabajar con varios ejemplos primero y después iniciar la escritura apoyados por el maestro y; cuando ya estén preparados hay que estimularlos para que trabajen de forma independiente. Veamos el siguiente ejemplo:

"Hay tres razones por las que me gusta la primavera. La primera es . La segunda es La última es

Estas son las tres razones por las que me gusta la primavera”.

Progresivamente se deben reducir las claves visuales de modo que los niños adquieran independencia en su trabajo y así ir progresando y mejorando los resultados. Unos pocos niños pueden desarrollar habilidades metacognitivas que les permita: supervisar el proceso de escritura, descubrir los errores y aplicar estrategias para corregirlos. Este grado de conciencia lectora y escritora es muy difícil de alcanzar en la mayoría de los niños y adolescentes $^{51}$.

\section{Deletreo}

El deletreo es una actividad que los chicos y las chicas con SXF ejecutan sin problema. Debido a que estos chicos están dotados de buena memoria visual para las palabras, se les facilita el recuerdo de cómo se deletrean y la reproducción que pueden hacer en el ordenador o de forma manual. Algunos de los SXF son capaces de usar el deletreo de palabras de forma parecida a como lo hacen otros niños en un aula regular. Sin embargo, muchos SXF necesitan trabajar el deletreo con listas de palabras adecuadas a su nivel cognitivo ${ }^{52}$. El deletreo debe incluirse como una de las metas de la lectura y la escritura.

Varias son las estrategias que ayudan a mejorar 
el deletreo. Estas estrategias para que sean eficaces deben apoyarse en las habilidades fuertes que poseen estos niños (sobre todo el procesamiento simultáneo visual) y reforzar aquéllas otras más débiles relacionadas con el procesamiento secuencial. Trabajar con actividades de rellenar espacios en blanco es una actividad que fortalece la memoria visual siempre que se acompañe de claves visuales parciales. Otra estrategia que también ayuda es que el maestro remarque el número de sílabas de las palabras, la secuencia de las letras, el número de letras ${ }^{53}$.

Finalmente, se recomienda la estrategia de usar sílabas incluidas en palabras deletreadas utilizando tiras de colores para después cortar la tira de cada una de las sílabas. Los chicos observan las partes de la palabra como si fuera un juego de sílabas revueltas durante un tiempo y de forma libre juegan un rato con las tarjetas y al final el maestro de lenguaje dicta la palabra muy despacio exagerando cada una de las sílabas y el niño tiene que unirlas para formar la palabra.

\section{Conclusiones}

Las alteraciones de lenguaje de los SXF constituyen una de las causas que explica alguna de las dificultades de lectura y escritura. Las alteraciones oscilan entre el tartamudeo leve y problemas graves en las habilidades lingüísticas básicas. Se estima que el $11 \%$ de los SXF a la edad de cinco años todavía no han iniciado el lenguaje oral ${ }^{54} \mathrm{y}$ aquéllos que lo han iniciado muestran cierta incapacidad para pronunciar las palabras de forma clara. Las dificultades práxicas, o de planificación motora, interfieren en la correcta articulación de los sonidos (fonéticos). El tratamiento en este aspecto puede llegar a ser especialmente difícil a causa de la hipersensibilidad sensorial en la zona oral ${ }^{55}$.

Otro aspecto que ha sido confirmado reiteradamente es la preferencia de estos niños por el procesamiento simultáneo de la información en lugar de secuencial. Este hecho repercute negativamente cuando la información que se debe procesar es de naturaleza verbal. Los SXF prelectores y lectores tienen serios problemas con muchas áreas auditivas: discriminar sonidos, reconocer sonidos fuera de las palabras, mezclar sonidos para construir palabras, silabear y reconocer si dos palabras riman. Estas generalizaciones son mucho más aplicables a los niños que a las niñas ${ }^{56,57}$. En efecto, las niñas manejan un vocabulario adecuado, aprenden palabras nuevas de forma rápida, reconocen los sonidos de manera precisa y usan la gramática de acuerdo a su edad.

En cuanto a la enseñanza y el aprendizaje de la lectura, se ha comprobado que la calidad ambiental, la instrucción de los profesores, la estructura de las aulas y la motivación son factores relevantes. Lo recomendable es fomentar aulas lo más normalizadas posibles, con maestros que se preocupen menos de los niveles funcionales y de los diagnósticos complejos y se ocupen más de diseñar estrategias que fomenten la adquisición y el desarrollo de habilidades lectoras. Estas habilidades deben incluir un primer nivel de reconocimiento de palabras y no-palabras, cortas y largas, regulares e irregulares, homófonos, etc. Sin embargo, el nivel de lectura de palabras es insuficiente y debería expandirse hacia un segundo nivel más funcional que abarcara tareas de comprensión. Para la obtención de este segundo nivel, existen algunas estrategias cuya eficacia ha sido comprobada con los SXF.

De manera similar, existen estrategias para el desarrollo de la escritura. Esta habilidad es de difícil adquisición para estos niños, pero no imposible. Un importante reto de los maestros es encontrar metodologías adecuadas y elementos de refuerzo que promuevan sensaciones placenteras con la actividad. El rechazo de la escritura suele estar asociado a dificultades motoras, muy frecuentes en los SXF, que producen disgrafías severas. La mayoría de los SXF requieren entrenamiento en estrategias kinestésicas como actividades previas al inicio formal de la escritura ${ }^{58}$. 


\begin{abstract}
Resumen
Introducción: El objetivo del artículo es realizar una revisión actualizada de las dificultades escolares de los niños y adolescentes con trastornos de X frágil. El síndrome X frágil (SXF) es la causa más frecuente de discapacidad intelectual hereditaria, así como una causa común de trastornos de aprendizaje y problemas conductuales. Se caracteriza por un fenotipo físico y un fenotipo conductual muy específicos. El SXF está causado por una mutación del gen FMR1 (Fragile X Mental Retardation), situado en la porción final del cromosoma X, locus Xq27.3. La mutación del gen produce un número anómalo de repeticiones de la tripleta CGG (CitosinaGuanina- Guanina). La mutación completa lleva consigo un estado de hipermetilación que inhibe la expresión del gen. El análisis del síndrome X frágil representa un buen modelo para determinar las relaciones entre genes y conducta. Desarrollo: El área de la lectura y la escritura en la población X frágil muestras demasiadas zonas oscuras. El número de trabajos de investigación básica y aplicada es escaso y mayoritariamente asume los patrones de comportamiento derivados de poblaciones próximas, como el sindrome Down, Autismo y sindrome Williams. Conclusión: Los principales problemas de los SXF con la lectura y la escritura tienen su origen en alteraciones de lenguaje y motoras y dificultades de integración sensorial. Nuestro trabajo pretende ampliar y sistematizar de manera orgánica algunos de los aspectos que consideramos fundamentales para mejorar la práctica educativa y la restauración de las deficiencias lectoras. En esta línea de actuación destacamos algunas estrategias metodológicas y propuestas programáticas que conviene explorar para conocer su valor pedagógico y su eficacia práctica.
\end{abstract}

Palabras clave: Síndrome X frágil, lectura, escritura, estrategias, aprendizaje, enseñanza, discapacidad intelectual.

\section{Referencias}

1. Martin J P. Bell J. A pedigree of mental defect showing sex linkage. J Neurol Neurosurg Psychiatry 1943; 6: 154-6.

2. Lubs H A. A marker X chromosome. Am J Hum Genet 1996, 21: 231-44.

3. Sutherland G R. Marker X chromosomes and mental retardation. N Engl J Medicine 1997; 296: 1415.

4. Crawford D C. Meadows K L. Newman J L, Talf L F, Scott E, Leslie M, et al. Prevalence of the fragile $\mathrm{X}$ syndrome in African-Americans. Am J Med Genet 2002; 110 (3): 226-33.

5. Hagerman R J. Medical follow-up and pharmacotherapy. En R J Hagerman \& P J Hagerman (Eds), Fragile $\mathrm{X}$ syndrome: Diagnosis, treatment and research. Baltimore: The Johns Hopkins University Press: 2002a.

6. Ferrando-Lucas M T, Banús-Gómez P, López Pérez G. Aspectos cognitivos y del lenguaje en niños con síndrome X frágil. Rev Neurol 2003; 36 (Supl 1): S137-42.

7. Ferrando-Lucas M T, Puente-Ferreras A. Niñas con síndrome $\mathrm{X}$ frágil: un modelo para los trastornos específicos del desarrollo. Rev Neurol 2008; 46 (Supl 1): S17-S19.

8. Hagerman P J, Hagerman R J. The fragile X premutation: A maturiting perspective. Am J Hum Genetics 2004; 74, 5: 805-16.

9. Maddalena A, Richardss C S, McGinniss M J, Brothhman A, Desnick R J, Grier R E, et al. Technical standards and guidelines for $\mathrm{X}$ fragile: The first of a series of disease-specific supplements to the 
Standards and Guidelines for Clinical Genetics Laboratories of the American College of Medical Genetics. Quality Assurance Subcommittee of Laboratory Practice Committee. Genet Medicine 2001; 3 (3): 2200-5.

10. Fu Y H, Kuhl D P A, Pizutti A, Pieretti M, Sutcliffe J S, Richards S, et al. Variation of the CGG repeat al the fragile $\mathrm{X}$ site results in genetic instability: resolution of the Sherman paradox. Cell 1991; 67: 1047-58.

11. Warren S T, Nelson D L. Advances in molecular diagnosis of fragile X syndrome. JAMA 1994; 271: 536-42.

12. Artigas-Pallares J, Brun-Gasca C. ¿Se puede atribuir el fenotipo conductual del síndrome X frágil al discapacidad intelectual y al trastorno por déficit de atención/hiperactividad? Rev Neurol 2004; 38 (1): 7-11.

13. Turner G, Webb T, Wake S, Robinson H. Prevalence of Fragile X Syndrome. Am J Med Genetics 1996; 64: 196-7.

14. De Vries B, Mohkamsing S, Van den Ouweland A M, Duivenvoorden H J, Mol E, Gelsema K, et al. Screening and diagnosis for the fragile $\mathrm{X}$ syndrome among the mentally retarded: an epidemiological and psychological survey. Collaborative Fragile X Study Group. Am J Hum Genetic 1997; 61: 660-7.

15. De Diego Otero Y, Hmadcha A, Carrasco Mairena M, Pintado Sanjuán E. Síndrome X frágil y discapacidad mental hereditaria. Madrid: Ed. Ministerio de Sanidad y Consumo: 1998.

16. Artigas-Pallarés J, Brun-Gasca C, Gabau E, Lahuerta A. Medical and neurological problems in fragile $\mathrm{X}$ syndrome. Results of a national questionnaire. 7th International Fragile X Conference. Los Ángeles: July 2000.

17. Buckley S, Johnson-Glenberg MC. Increasing literacy learning for individuals wit Down syndrome and fragile X syndrome. En J. E. Roberts, R. S. Chapman \& S. F. Warren (Eds), Speech and language development and intervention in Down and Fragile X syndrome (pp. 193-218). Baltimore. Paul H. Brookes Publishing Co: 2008.

18. Groen M, Laws G, Nation K, Bishop D V M. A case of exceptional reading accuracy in a child with
Down syndrome: Underlying skills and the relation to reading comprehension Cogn Neuropsych C 2006; 23 (8): 1190-214.

19. Johnson-Glenberg M C. FXS syndrome: Memory skills and the emergence of reading in males. En R. Paul (Ed), Language disorders from a developmental perspective: Essays in Honor of Robin Chapman. Mahwah, NJ: LEA: 2007a.

20. Abbeduto L, Hagerman R J. Language and communication in fragile $\mathrm{X}$ syndrome. Ment Retard Dev Disabil Res Rev 1997; 3: 313-22.

21. Benetto L, Penington B F. The neuropsychology of fragile X syndrome. En R J Hagerman \& A C Cronister (Eds), Fragile X syndrome: Diagnosis treatment, and research (pp. 210-248). Baltimore: The Johns Hopkins University Press: 2002.

22. Bailey D B, Nelson D. The nature and consequences of fragile X syndrome. Ment Retard Dev Disabil Rese Rev 1995; 1: 238-44.

23. Bailey D B. Jr, Hatton D D, Mesibov G, Ament N, Skinner M. Early development, temperament, and functional impairment in autism and fragile $\mathrm{X}$ syndrome. J Autism Dev Disord 2000; 30 (1): 49-59.

24. Braden M L. Fragile, handle with care: More about fragile X syndrome, adolescents and adults. Dillon, CO: Spectra Publishing Co: 2000.

25. Roberts J E, Long S H, Malkin C, Barnes E, Skinner $\mathrm{M}$, Hennon E A, et al. A comparison of phonological skills of young boys with fragile $\mathrm{X}$ syndrome and Down syndrome. J Speech Lang Hear Res 2005; 48 (5): 980-95.

26. Symons F J, Clark R D, Roberts J P, Bailey D B. Classroom behaviour, and academic engagement of elementary school-aged boys with fragile $\mathrm{X}$ syndrome. J Spec Educ 2001; 34 (4): 194-202.

27. Braden M L. Formal assessment and constructing an individual programme. En D. Dew-Hughes (Ed), Educating children with fragile X syndrome (pp. 61-72). London: Routledge Farmer: 2004.

28. Johnson-Glenberg M C. FXS syndrome: A neuronal network model of sequential memory in males. Cog Syst Res 2008; 9 (4): 274-92.

29. Roberst J E, Chapman R S, Warren S F. Speech and language development and intervention in Down and Fragile X syndrome. Baltimore. Paul H. Brookes Publishing Co: 2008. 
30. Johnson-Glenberg M C. FXS syndrome: Memory skills and the emergence of reading in males. En R. Paul (Ed), Language disorders from a developmental perspective: Essays in Honor of Robin Chapman. Mahwah, NJ: LEA: 2007a.

31. Braden M L. Mujeres afectadas por el síndrome X frágil. En F J Ramos (Ed), El síndrome X frágil: Material educativo de la Fundación Nacional de los Estados Unidos: 1999.

32. Johnson-Glenberg M C. Literacy and working memory in those with FXS syndrome. Poster session presented at Symposium on Research on Children's Language Disorders, Madison. WI: 2003.

33. Roberts J, Hennon A A, Anderson K. FXS syndrome and speech and language. The ASHA Leader 2003; 8 (19): 6-10.

34. Saunders S. Fragile X syndrome: A guide for teachers. London: David Fulton Publisher: 2001.

35. Valett R E. Dislexia. Barcelona: CEAC: 1992.

36. Stackhouse T M. Conceptos de integración sensorial y el síndrome X frágil. En F. J. Ramos Fuentes (Ed.), El síndrome X frágil. Madrid: Ministerio de Trabajo y Asuntos Sociales: 1999.

37. Johnson D, Myklebust H. Learning disabilities: Educational principles and practices. NY: Grune and Station: 1967.

38. Woodcock R W. Woodcock reading mastery test revised-normative update (WMRT-R/UN). Circle Pines, MN: American Guidance Service: 1998.

39. Thorndike R L, Hagen E P, Sattler JM. StanfordBinet intelligence scale ( $4^{\text {th }}$ ed.). Itasca, IL: Riverside: 1986.

40. Siegel L S. Phonological processing deficits as the basis of a reading disability. Dev Rev 1993; 13: 246-57.

41. Connors F A, Atwell J A, Rosenquist C J, Sligh A C. Abilities underlying decoding differences in children with intellectual disability. J Intellect Disabil Res 2001; 45: 292-9.

42. Byrne B. The foundation of literacy: The child's acquisition of the alphabetic principle. Hove, England: Psychology Press: 1998.

43. Byrne B, Fielding-Barnsley R, Asheley L. Effects of phoneme identity training after six years: Outocome level distinguished from rate of response. J Edu Psychol 2000; 92: 659-67.
44. Kumin L. Classroom language skills for individuals with Down syndrome: A guide for parents and teachers. Bethesda, MD: Wooddbine House: 2001a.

45. Harris-Schmidt G. Characteristics of speech language disorders in children with fragile $\mathrm{X}$ syndrome. Retrieved February 6, 2006, from http://www. Fragilex.org/html/cognitive.htm

46. Gathercole S E, Pickering S J. Working memory deficits in children with special educational needs. Brit J Spec Edu 2001; 28 (2): 89-97.

47. Fowler A E. The challenge of linguistic mastery in Down syndrome. En T S Hassold (Ed), Down syndrome: A promising future, together (pp. 165182). New York: Wiley-Liss: 1999.

48. Ronald J A. Edwards S. Language in mental retardation. London: Whurr: 1997.

49. Abbeduto L, Brady N, Kover S. Language development and fragile $\mathrm{X}$ syndrome: Profiles, syndrome specificity, and within-syndrome differences. Ment Retard Dev Disabil Res Rev 2007; 13: 36-46.

50. Kumin L, Council C, Goodman M. The pacing board: A technique to assist the transition from single word to multiword utterances. Inf-Todd Interv 1995; 5: 23-9.

51. Puente A, Jiménez V, Alvarado J M. Escala de conciencia lectora (ESCOLA). Madrid: Editorial EOS: 2009.

52. Kumin L. Language intervention to encourage complex language use. En J E Roberts, R S Chapman \& Warren, S. F. (Eds), Speech and language development and intervention in Down and Fragile X syndrome (pp. 193-218). Baltimore. Paul H. Brookes Publishing Co: 2008.

53. Bryant P, Bradley L. Problemas infantiles de lectura. Madrid: Alianza Editorial: 1998.

54. Taylor R, Scharfenaker S, O’Connor R, Lampe M, Kovach T, Hills J, et al. Severe language impairment in fragile X syndrome: clinical correlates and treatment approaches. Paper presented at the $5^{\text {th }}$ International Conference of Fragile X and Xlinked Mental Retardation, 1998, Asheville, North Carolina, July 2629.

55. Brun Gasca C, Artigas Pallarés J. Aspectos psicolingüísticos en el síndrome del cromosoma $\mathrm{X}$ frágil. Rev Neurol 2001; 33 (Supl 1): S 29-S32.

56. Ramos Fuentes F. Fenotipo físico y manifestacio- 
nes clínicas. En Ma Isabel Tejera Minguez (Ed), Síndrome X Frágil. Madrid, Ministerio de Trabajo y Asuntos Sociales: 2006.

57. Brun-Gasca B. Fenotipo cognitivo-conductual. En $M^{a}$ Isabel Tejera Miguez (Ed), Síndrome X Frágil. Madrid, Ministerio de Trabajo y Asuntos Sociales:
2006.

58. Rigau E, García Novell K. Tratamientos psicológicos, conductuales y logopédicos en la primera infancia y hasta la adolescencia. En Ma Isabel Tejera Minguez (Ed), Síndrome X Frágil. Madrid, Ministerio de Trabajo y Asuntos Sociales: 2006.

\section{Correspondencia:}

Aníbal Puente F.

E-mail: apuente@psi.ucm.es 